\title{
Design on Heterogeneous Seamless Integration of Wireless Data Acquisition System
}

\author{
Wang Shenghui \\ Institute of Electric and Electronics Engineering \\ Changchun University of Technology \\ Changchun, China
}

\author{
Jiang Changhong \\ Corresponding Author \\ Institute of Electric and Electronics Engineering \\ Changchun University of Technology \\ Changchun, China
}

\author{
Jin Xing \\ Institute of Electric and Electronics Engineering \\ Changchun University of Technology \\ Changchun, China
}

\begin{abstract}
Based on wireless sensor network technology, this paper focuses on the study of the problem of heterogeneous seamless integration of multi-sensor nodes and puts forward a correlation protocol concerning SMAC multi-point, multi-type sensor network nodes self-organizing wireless networking application. This technology has been used in the energy source information acquisition system of a concentrating mill, which has realized 738 site collecting points, 6 types of sensor nodes, the real-time acquisition of 8 types of power information and 6 types of additional information, and has constructed a heterogeneous wireless network platform of information sharing and interactive control.
\end{abstract}

Keywords-Wireless sensor network, Heterogeneous seamless integration, Data acquisition system

\section{INTRODUCTION}

A stable and reliable data acquisition system is the basis of the monitoring, instrument fault diagnosis and energy source information management in large-scale industry control systems. The current data acquisition systems are mainly based on OSI/RM model, TCP/IP protocol and CAN bus. The development tendency of control diversification and that of system decentralization expose gradually the weakness of OSI/RM in real-time data acquisition of various types of monitoring points, the robustness and the inadequacy of safety of data acquisition and transmission under complex control commands in TCP/IP and the defect in the interconnection between CAN and Internet. Wireless sensor network technology provides a new approach to solving this kind of problems. Moreover, it is characterized by high performance, low cost, no need of site wiring, small alteration of instruments and devices, convenient and fast operation and other advantages and is particularly applicable to the data acquisition system in large-scale industrial districts.

Based on wireless sensor network technology, the signal extraction of sensor nodes in multi-point and multi-type data acquisition system is studied in this paper, and a network protocol based on SMAC heterogeneous seamless integration is put forward, which provides efficient and stable monitoring system fore-end technology for the realtime data acquisition, the control and management of wireless transmission as well as the information integration of the heterogeneous industrial control system of a large number of different types of sensor nodes .

\section{HeterogeneOUS SEAMLESS INTEGRATION BASED ON}

\section{WIRELESS SENSOR NETWORKING}

Wireless sensor network is a technology of data acquisition, transmission and processing, and is used in the monitoring and control field in a self-organizing wireless networking coordinative fashion. This technology, initiated by Professor William J. Kaiser, is a miniature wireless node network designed for automatic sensing of information of the monitoring area. Due to the adoption of the datacentered, high-density and multi-hop network transmission technology, wireless sensor network becomes independent and stable in the real-time acquisition and transmission of large-scale monitoring information. Nonetheless, when it is faced with the information integration of heterogeneous industrial control systems, the conflict between various protocols, wireless communication disturbance and some other problems emerge inevitably. Therefore, we have customized a network protocol with application correlation based on SMAC protocol and have realized the seamless integration of heterogeneous information exchanges from the site instrument layer to the process monitoring layer, which have facilitated the data transmission, storage and sharing between different monitoring systems[2-5].

The integration of wireless sensor network with heterogeneous seamless integration expands in two aspects the multi-point [6], multi-type information monitoring and data acquisition system. The first one is the expansion of the system frame structure, which makes breakthroughs in the wired system modes such as the existing OSI/RM model, TCP/IP protocol, CAN bus of data acquisition system, and constructs a wireless network, real-time intelligent information acquisition platform, decreases the complexity of transmission path and enhances the expandability and maintainability of the architecture. The other one is the expansion of information diversification. The embedment of heterogeneous seamless integration technology into the data acquisition system makes it capable of information sharing 
and interoperability, which facilitates the analysis and mining of data and provides comprehensive decision support.

\section{NetWork Structure OF THE System}

The multi-point, multi-type information monitoring and data acquisition system consists of four major links of data acquisition, processing, transmission and intelligent information integration. It introduces wireless sensor network and the technology of heterogeneous seamless integration, constructs a dynamic, real-time, omnidirectional, multivariable and complex data acquisition system using wireless sensor network as the core. Its structure is shown in Figure 1.

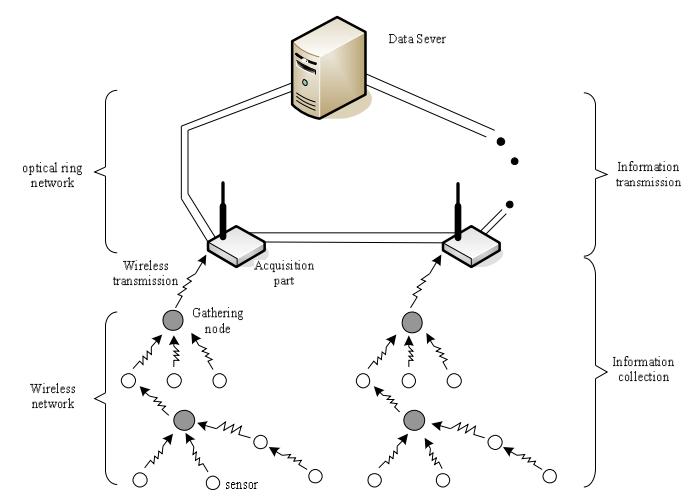

Figure 1. Network structure of data acquisition system

The network structure of the data acquisition system comprises three layers from the bottom up. The first is information acquisition layer, composed of wireless sensor intelligent nodes which distribute within the monitoring area, form a wireless network in a self-organizing fashion and collect the site data and information real time. The second is information transmission layer, composed of sink nodes and collectors. The collected information is uploaded by sensor nodes to sink nodes and then transmitted from there to collectors and finally to the servers of information centre by the collectors using optical ring network. The third is information management layer, composed of servers, which complete the management and control of the integration of data and information, command issuing, storage and backup, etc..

\section{A. Components of Network Nodes}

Each wireless sensor network intelligent node serves as an intelligent unit of the acquisition system of monitoring data, adopts independently embedded MCS08GT60 single chip and possesses built-ins 4KRAM, 64KFLASH and 10BitADC. It can extend 512MB fast RAM, and has outside extended bus interfaces such as $\mathrm{I}^{2} \mathrm{C}$, SCI TX $\backslash \mathrm{RX}$, GPIO, TPM, IRQ, RS232, RS485 and USB, and can connect various types of sensors. It supports $40 \mathrm{MHz}$ external clock and can be connected to MC13193 by SPI interface. Based on the standard for wireless individual regional network communication, the highest transmission rate of data acquisition for single sensor node can be $250 \mathrm{Kbps}$ at 2.4GHz[7].

\section{B. Heterogeneous Seamless Integration Protocol}

Due to the differences in the types of the monitoring objects within the working area for the multi-point, multitype information monitoring and data acquisition system characterized by regional dispersion and load dispersion, the collected data and information by the sensor nodes inevitably differentiate in their types and communication protocols. For example, in the monitoring system of power quality information, the measurement of electricity quantity, the monitoring of current and voltage and the measurement of the parameters such as power factor and harmonics can be achieved by Weisheng meter, motor synthetical protector, ACR or PDM series of monitoring instruments for electric parameters, etc., but the communication protocols of electric instruments of different models and from different manufacturers are different from each other. To realize the real-time acquisition and transmission of data of various types of sensor nodes in the wireless sensor network, a network protocol based on SMAC heterogeneous seamless integration is put forward, which is shown in Table I.

The definition format of monitoring command data packet of sensor nodes consists of five parts, namely protocol type, base station address (source address), destination task and meter type. According to the instrument type of the data packet and the type of the executed task, a matched protocol type is chosen and the destination address of sensor nodes is identified, and site data and information are collected, such as three-phase voltage, three-phase current, active power, reactive power and power factor[8].

Based on this protocol, command operations independent of sensor node signal acquisition in the data acquisition system can be carried out; and the interface to application program objects, the representation interface to macrolanguage objects and the sharing data interface to the application programs between different monitoring systems can be provided. On the basis of these interfaces, wireless sensor network can be opened to and interconnected with other monitoring systems so as to realize the information sharing and processing of the monitoring system.

\section{POWER INFORMATION DATA ACQUiSITION System}

Power information data acquisition system have monitored the power information such as three-phase voltage, three-phase current, normal-phase active, reactivephase active, active power, reactive power, power factor and frequency, of the 738 sampling points distributed over 7 regions of a concentrating mill covering an area of 30000 square meters by adopting the frame structure of wireless sensor network in combination of the network protocol based on SMAC heterogeneous seamless integration. Figure 2 is the site data acquisition platform of power information data acquisition system.

The site instruments in Figure 2 can be arranged according to the monitoring command information of sensor nodes issued by the data processing center and also be 
altered by the operation personnel. Figure 4 is the site data acquisition platform. These data originate from 6 different types of sensor nodes. In addition to the information of necessary power parameters, additional information is included as well, such as instrument name and type, acquisition time, sampling frequency, digits of data, check digit, etc.. There are 738 site collection points, and the realtime data acquisition, data sharing and access control can be realized in the constructed wireless network acquisition system.

The energy source information acquisition system of the concentrating mill concentrates on the acquisition, processing and integration of power information and energy consumption information in various production processes such as crushing, magnetic separation, tailings and pelletizing. Since its operation in July up to the present, the system runs smoothly and is stable in the data acquisition, storage and control. The results indicate that the designed data acquisition system is capable of stable data acquisition and adaptable to harsh environment. The integration of wireless sensor network with heterogeneous seamless integration makes possible the real-time data acquisition, wireless transmission control and management as well as the information integration of the heterogeneous industrial control system of a large number of different types of sensor nodes.

\section{CONCLUSION}

In this paper, the design and realization of multi-point, multi-type information monitoring and data acquisition system have been studied from the aspects of system structure and heterogeneous seamless integration. The customized heterogeneous network protocol based on SMAC has completed the software and hardware design of wireless sensor network intelligent nodes and has been applied in the energy source information data acquisition system of a concentrating mill and has realized the data acquisition of more than 700 points of six types.

\section{ACKNOWLEDGMENT}

The project is Supported by National Science and Technology Foundation of China (2007BAE17B03).

\section{REFERENCES}

[1] Flammini; Ferrari P; Sisnni E, et al. Sensor integration in industrial environment: from fieldbus to web sensors. Computer Standards \& Interface [J], 2003.25(2), PP183-194.

[2] S. A. Aldosari J; M. F. Moura. Detection in decentralized sensor networks. Proc. IEEE International Conference on Accoustics, Speech, and Signal Processing, 2004.5(2), PP 277-280.

[3] Liming; Sun etc. Wireless Sensor Network, Beijing Tsinghua University Publishing, 2005.

[4] H. Wang; J. Elson; L. Girod; et al. Target classification and localization in habitat monitoring. Proc. IEEE International Conference on Acoustics, Speech, and Signal Processing. 2003.4(4), PP844-847.

[5] F. Akyildiz, W. Su, Y. Sankarasubramaniam, and E. Cayirci, "A survey on sensor networks," IEEE Commun. Mag., Aug. 2002, pp. 102-114.

[6] Desjardins S L; Londono N A; Lau D T, et al. Real-time data processing and graphical user interface (GUI) for continuous structural monitoring. Proceeding of Developments in Mechanics of Structures and Materials. Perth, Australia, 2004, PP1191-1196.

[7] Y. Yuan; M. Kam. Distributed decision fusion with a random-access channel for sensor network applications. IEEE Trans. Instrum. Meas. 2004.4(53), PP1339-1344

[8] A. D'Costa; A. M. Sayeed. Data versus decision fusion in sensor networks. Proc. IEEE International Conference on Acoustics, Speech, and Signal Processing. 2003.4(4), PP 832-835.

TABLE I. THE DEFINITION FORMAT OF MONITORING COMMAND DATA PACKET OF SENSOR NODES

\begin{tabular}{|c|c|c|c|c|}
\hline \multirow{3}{*}{$\begin{array}{l}\text { Protocol } \\
\text { Type }\end{array}$} & \multirow{3}{*}{$\begin{array}{c}\text { Base Station } \\
\text { Address }\end{array}$} & \multicolumn{3}{|c|}{ Destination } \\
\hline & & Target Address & & Meter Type \\
\hline & & \multicolumn{3}{|c|}{ Task } \\
\hline $0 \times 01$ & $0 \times 10$ & $0 \times 01$ & 0xC79AC000 & DSSD331-I \\
\hline $0 \times 02$ & $0 \times 20$ & $0 \times 6800000000000168$ & $0 \times 43 \mathrm{C} 3$ & DSSD331-II \\
\hline $0 \times 03$ & $0 \times 30$ & $0 \times 01$ & 0x030028000F & Motor Protector \\
\hline $0 \times 04$ & $0 \times 40$ & $0 \times 01$ & 0x0301A10016 & PDM803 \\
\hline $0 \times 05$ & $0 \times 50$ & $0 \times 01$ & 0x0301000020 & PDM820 \\
\hline $0 \times 06$ & $0 \times 60$ & $0 \times 01$ & 0x0301000025 & ACR320E \\
\hline
\end{tabular}




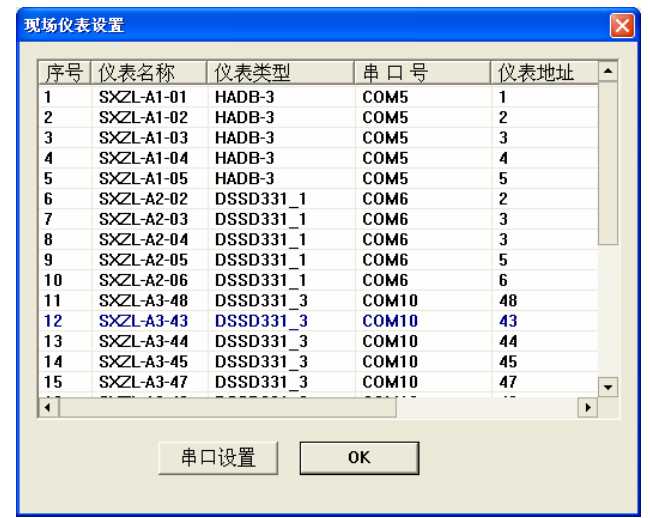

\begin{tabular}{|c|c|c|c|c|c|c|c|c|c|c|}
\hline Instrunet_Hame| & Record_Time & Voltage_A & Yoltage_B & \begin{tabular}{l|l} 
Voltage_C & \\
\end{tabular} & Current_A & Current_B & \begin{tabular}{|l|} 
Current_c \\
\end{tabular} & 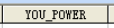 & WU_POWER & Plaz_ 1 \\
\hline + QDGI_A1_02 & $2009-7-28 \quad 12: 10: 04$ & 60.31 & 60.58 & 80.63 & .089 & .089 & .0845 & 12774.577 & 16330.08820833 & \\
\hline + QDGY_A1_03 & $2009-7-28 \quad 12: 10: 10$ & 60.1 & 60.69 & 60.66 & 2385 & 2305 & 2295 & 52069.66991667 & 65770.10545833 & \\
\hline + QDGY_A1_04 & $2009-7-28 \quad 12: 10: 15$ & 60.35 & 60.55 & 60.62 & .073 & 0645 & .062 & 45939.021125 & 76508.83516667 & \\
\hline + QDGY_A1_05 & $2009-7-28 \quad 12: 10: 21$ & 60.3 & 60.48 & 60.52 & .0715 & 0655 & .0595 & 62931.95941667 & 66520.01066667 & \\
\hline + QDGY_A1_06 & $2009-7-28 \quad 12: 10: 26$ & 60.31 & 60.58 & 60.55 & .944 & .9245 & .9245 & 97327.44625 & 116857.288125 & \\
\hline + QDGI_A1_07 & $2009-7-28 \quad 12: 10: 32$ & 60.3 & 60.61 & 60.58 & 2.3155 & 2.3095 & 2.3305 & 73870.58341667 & 175364.2431667 & \\
\hline + QDGI_A1_08 & $2009-7-28 \quad 12: 10: 38$ & 60.26 & 60.66 & 60.56 & .8655 & .8655 & .8835 & 45430.889625 & 72538. 35958333 & \\
\hline + QDGI_A1_09 & $2009-7-28 \quad 12: 10: 43$ & 60.19 & 60.44 & 60.44 & 6.545 & 6.44 & 6.53 & 104004.8980417 & 73205.15483333 & \\
\hline + QDGI_A1_10 & $2009-7-28 \quad 12: 10: 49$ & 60.23 & 60.53 & 60.51 & 1.7445 & 1.7675 & 1.8065 & 193211.1780417 & 151388.872375 & \\
\hline + QDGI_A1_11 & $2009-7-28 \quad 12: 10: 55$ & 60.21 & 60.45 & 60.48 & 1.5205 & 1.514 & 1.5235 & 84037.92341667 & 83762.397875 & \\
\hline + QDGI_A1_12 & $2009-7-28 \quad 12: 11: 00$ & 60.31 & 60.59 & 60.6 & 1.0.0 & 0 & 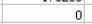 & 0 & 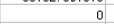 & \\
\hline + QDGI_A1_18 & $2009-7-28 \quad 12: 11: 06$ & 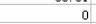 & 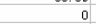 & 年 & 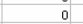 & 0 & 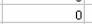 & 0 & 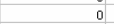 & \\
\hline + QDGY_A1_19 & $2009-7-28 \quad 12: 11: 11$ & 60.05 & 60.62 & 60.6 & 1.914 & 1.8645 & 9635 & 111868.23175 & 63100.97945833 & \\
\hline + QDGY_A1_20 & $2009-7-28 \quad 12: 11: 17$ & 60.07 & 60.62 & 60.62 & 68 & .666 & .7065 & 111234.5524583 & 66787. 44729167 & \\
\hline+ QDGI_A1_21 & $2009-7-28 \quad 12: 11: 23$ & 60.39 & 60.65 & 60.68 & .688 & .6745 & .715 & 213752.6439167 & 118152.33925 & \\
\hline + QDGI_A1_22 & $2009-7-28 \quad 12: 11: 28$ & 60.47 & 60.71 & 60.7 & 0 & 0 & & 122422. 9756667 & 82940.22708333 & \\
\hline+ QDGY_A1_23 & $2009-7-28 \quad 12: 11: 34$ & 60.2 & 60.74 & 60.76 & 0 & 0 & 0 & 130892.658125 & 93675.53370833 & \\
\hline + QDGY_A1_24 & $2009-7-28 \quad 12: 11: 40$ & 60.44 & 60.72 & 60.67 & 0 & 0 & & 39481.62820833 & 98430.53120833 & \\
\hline + QDGY_A1_25 & $2009-7-28 \quad 12: 11: 45$ & 60.38 & 60.65 & 60.62 & 0 & 0 & & 17229.08295833 & 48300.09145833 & \\
\hline + QDGY_A1_2B & $2009-7-28 \quad 12: 11: 51$ & 60.36 & 60.63 & 60.65 & 1.7125 & 1.658 & 1. 7635 & 113357.0749583 & 73528.689125 & \\
\hline+ QDGI_A1_27 & $2009-7-28 \quad 12: 11: 56$ & 60.35 & 60.59 & 60.63 & 1.766 & 1.812 & 1.7145 & 2321.767416667 & 3564.183625 & \\
\hline + QDGY_A1_28 & $2009-7-28 \quad 12: 12: 02$ & 60.35 & 60.57 & 60.57 & 1.1215 & 0 & 1.122 & 29344.25133333 & 40742.178 & \\
\hline+ + QDGY_A1_29 & $2009-7-28 \quad 12: 12: 08$ & 60.13 & 60.67 & 60.66 & 2.264 & 2.2085 & 2.3275 & 148416. 6189583 & 112073.9630417 & \\
\hline + QDGY_A1_30 & $2009-7-28 \quad 12: 12: 13$ & 60.3 & 60.59 & 60.61 & .7665 & .786 & .7535 & 1821.843208333 & 2294.40725 & \\
\hline + QDGY_A1_34 & $2009-7-28 \quad 12: 12: 19$ & 60.3 & 60.59 & 60.58 & .0915 & .0765 & .094 & 31125. 39308333 & 57922.44966667 & \\
\hline + QDGI_A1_35 & $2009-7-28 \quad 12: 12: 25$ & 60.27 & 60.57 & 60.55 & 0 & 0 & & 80912.38891667 & 186431.1831667 & \\
\hline + QDGY_A1_36 & $2009-7-28 \quad 12: 12: 30$ & 60.34 & 60.63 & 60.61 & .574 & .559 & .564 & 79879.360375 & 93719. 33516667 & \\
\hline + QDGI_A1_37 & $2009-7-28 \quad 12: 12: 36$ & 60.31 & 60.58 & 60.55 & 152 & .1455 & 1425 & 45729.82858333 & 34615.353375 & \\
\hline + QDGI_A1_38 & $2009-7-28 \quad 12: 12: 41$ & 60.29 & 60.56 & 60.53 & .074 & .062 & .0585 & 24609.976375 & 28484. 97204167 & \\
\hline + QDGI_A1_39 & $2009-7-28 \quad 12: 12: 47$ & 60.25 & 60.54 & 60.53 & .0705 & .7645 & 7675 & 106188.372875 & 63842.40258333 & \\
\hline + QDGI_A1_40 & $2009-7-28 \quad 12: 12: 53$ & 60.08 & 60.38 & 60.32 & 18.855 & 19.175 & 19.34 & 219760.7134583 & 161009.236125 & \\
\hline + QDGI_A1_41 & $2009-7-28 \quad 12: 12: 58$ & 60.18 & 60.43 & 60.44 & .5475 & .5565 & 544 & 26303.50841667 & 48071.73395833 & \\
\hline + QDGI_A1_42 & $2009-7-28 \quad 12: 13: 04$ & 59.83 & 60.4 & 60.37 & 0 & 0 & 0 & & .0025833333333 & \\
\hline + QDGI_A1_43 & $2009-7-28 \quad 12: 13: 10$ & 60.24 & 60.51 & 60.48 & 0 & 0 & 0 & 101631.258375 & 92069.45891667 & \\
\hline + QDGY_A1_50 & $2009-7-28 \quad 12: 13: 15$ & 59.91 & 60.61 & 60.61 & 0 & 0 & 0 & 933. 3465416667 & 2394.59425 & \\
\hline + QDGI_A1_51 & $2009-7-28$ 12:13:21 & 60.18 & 60.47 & 60.38 & 0 & 0 & 0 & 385.4658333333 & 1184.048458333 & \\
\hline
\end{tabular}

Figure 2. Site data acquisition platform 